\title{
Extraction of Signals Buried in Noise: Non-Ergodic Processes
}

\author{
Nourédine Yahya Bey \\ UFR des Sciences, Département de Physique, Université François Rabelais, Tours, France \\ E-mail: nouredine.yahyabey@phys.univ-tours.fr \\ Received April 15, 2010; revised August 19, 2010; accepted October 2, 2010
}

\begin{abstract}
In this paper, we propose extraction of signals buried in non-ergodic processes. It is shown that the proposed method extracts signals defined in a non-ergodic framework without averaging or smoothing in the direct time or frequency domain. Extraction is achieved independently of the nature of noise, correlated or not with the signal, colored or white, Gaussian or not, and locations of its spectral extent. Performances of the proposed extraction method and comparative results with other methods are demonstrated via experimental Doppler velocimetry measurements.
\end{abstract}

Keywords: Buried Signals, Stationary Non-Ergodic Processes, Spectral Analysis, White Noise, Colored Noise, Correlated Noise, Doppler Velocimetry

\section{Introduction}

Quality of signal information in different areas of science is degraded by encountered various natures of noises. This degradation may take different forms and evolves to observations where the time-averaged correlation function of a process is different from the ensemble-averaged function [1,2]. Non-linear filtering and their corresponding asymptotic stability are generally proposed to handle such processes [3-5].

In situations where signals are totally buried in noise and for which no a-priori information is available, ergodic hypothesis cannot be validated. Moreover, none of the above methods is suitable for extraction of such buried signals. In real world situations, non-ergodic processes in which a desired signal is buried may occur as shown by Doppler velocimetry measurements [6-11] used in this work.

It is crucial to notice that by the terms extraction of signals, we mean extraction of clean spectra of buried signals in noise and by buried signals, we mean signals defined for low or extreme low signal-to-noise ratio. In [12,13], we proposed two equivalent extraction methods, called respectively "modified frequency extent denoising (MFED)" and "constant frequency extent denoising (CFED)". For easy reference, let us recall that the frequency extent is the interval $\left[0, f_{e}\right]$ where $f_{e}$ is the sampling frequency of the buried continuous-time signal to be extracted. The first procedure (MFED) is based on modifying the sampling frequency and the second one (CFED) is suited to a collection of available sample noisy processes for which the sampling frequency is constant. Clearly, CFED offers implementation simplicity since in most applications, signals are defined for a constant sampling frequency. Proposed extraction does not use any a-priori information on the signal to be extracted, works without averaging or smoothing in the direct time or dual frequency space, and it is achieved independently of the nature of noise (colored or white, Gaussian or not) and locations of its spectral extent.

Extraction methods in [12,13] are based on the theory of ergodic stationary processes. Extension of these results to extraction of signals correlated with noise in which they are buried are reported in [14]. We have shown that extraction of signals correlated with noise is achieved without averaging and independently of the nature of noise (correlated or not, correlated or not with the signal, colored or white, Gaussian or not) and locations of its spectral extent.

In this work, we extend results of one of the aforementioned extraction methods (CFED), to a more general case where ergodic hypothesis, given experimental conditions, cannot be validated, which is a fact and an issue. 
In Section 2, we recall principal definitions and results of the CFED extraction method since this method offers implementation simplicity as mentioned above. In Section 3, expression of extracted spectra of buried signals from non-ergodic processes is derived without any sort of averaging or smoothing in the time or frequency domain and without assuming the signal uncorrelated with noise. Extraction performances and comparative results with other methods [15-18] are discussed and illustrated in Section 4. It is shown that CFED extracts the Doppler mean frequency from experimental Doppler velocimetry measurements for which ergodic hypothesis is not validated or satisfied.

\section{Fundamentals $[12,13]$}

In this section, we recall some principal results reported in $[12,13]$.

\subsection{Signal Representation}

Let us consider a finite observation $z_{T}(t)$ of $z(t)$, a process containing a band-limited signal buried in zero-mean wide sense stationary noise $b(t)$, in the interval of length $T$ with $T f_{\max } \gg 1$. The process $z_{T}(t)$, available at the output of a low-pass filter of cut-off frequency $f_{\max }$, is given by,

$$
z_{T}(t)=\left\{\begin{array}{cc}
b_{T}(t)+s_{T}(t), & t \in[0, T] \\
0, & \text { otherwise, }
\end{array}\right.
$$

where $b_{T}(t)$ and $s_{T}(t)$ represent respectively the additive noise (white or colored) and the signal observed in the time interval of length $T$.

By considering the instants $t_{n}=n / f_{e}$ where $f_{e} \geq 2 f_{\max }$ is the sampling frequency, we can define the discretetime process $z_{N}(n)$ with $N=T f_{e}$.

\subsection{The Sample Power Spectral Density (SPSD) [12]}

Given $\left\{z_{N}(0), z_{N}(1), \cdots, z_{N}(N-1)\right\}$, we can form the estimate,

$$
\Phi\left(f, f_{e}, T\right)=\left|(1 / T) \operatorname{DFT}\left(z_{N}(n)\right)\right|^{2},
$$

where $\operatorname{DFT}\left(z_{N}(n)\right)$ denotes Discrete Fourier Transform of $z_{N}(n)$. Here the estimate $\Phi\left(f, f_{e}, T\right)$ depends on the frequency, $f$, the sampling frequency, $f_{e}$, and the length of the observation interval, $T$.

It is crucial to notice that (2) is not a power spectral density in the usual sense. In [12], (2) is defined as the "Sample" power spectral density or the sample spectrum of the process $z_{N}(n)$. In the following, we recall for easy reference the CFED extraction method.

\subsection{CFED Extraction Method}

Here, we have a collection of $\beta$ realizations of duration $T$ of a noisy process so that the length of the total observation interval is $\beta T$. These $\beta$ realizations denoted $z_{T}^{(p)}(t)$ where $p=0, \cdots, \beta-1$ are now concatenated, i.e.,

$$
z_{\beta T}(t)=\sum_{p=0}^{\beta-1} z_{T}^{(p)}(t-p T)
$$

\subsubsection{Sample Spectrum of Noise}

We found that the sample spectrum of noise obtained by Fourier transformation of (3) is given by [12],

$$
\phi\left(f, f_{e}, \beta T\right)=\sum_{p=0}^{\beta-1} \alpha_{p}(\beta) \phi\left(f-p /(\beta T), f_{e}, T\right),
$$

where $\phi\left(f-p /(\beta T), f_{e}, T\right)$ are translated copies of the original sample spectrum of noise whose components are spaced with the mutual distance $1 / T$ on the frequency axis.

It is crucial to notice that $\alpha_{p}(\beta)$ in (4) are reduction factors defined by,

$$
\sum_{p=0}^{\beta-1} \alpha_{p}(\beta)=1
$$

For the sake of simplicity and without loss of generality, we let,

$$
\forall p=0, \cdots, \beta-1, \alpha_{p}(\beta)=1 / \beta .
$$

Notice that a justification of (6) is found in [14]. Factors $\alpha_{p}(\beta)$ reduce indifferently translated copies the original sample spectrum of noise independently of their nature (white or colored, Gaussian or not) and act indifferently at all frequencies.

\subsubsection{Spectral Distribution}

Spectral lines of each copy of the original sample spectrum of noise $\phi\left(f-p /(\beta T), f_{e}, T\right)$ of noise are separated by the mutual distance $1 / T$. As these copies are shifted by $1 /(\beta T)$ with respect to each other (see (4)), the resulted sample spectrum $\phi\left(f, f_{e}, \beta T\right)$ will exhibit spectral lines separated by the mutual distance $1 /(\beta T)$. Hence original spectral lines of noise separated by the mutual distance $1 / T$ are now distributed in new $\beta$ frequency locations created in each frequency interval of length $1 / T$.

On the other hand, the spectrum of the signal $s_{T}(t)$ as given by the transformation of concatenated realizations (3), is specified by $\Gamma\left(f, f_{e}, \beta T\right)$. Since $\beta$ zeros are distributed in $\beta$ frequency locations created in each interval of length $1 / T$ (see [12]) then, 


$$
\Gamma\left(f, f_{e}, \beta T\right)=\Gamma\left(f, f_{e}, T\right) .
$$

\subsubsection{Extraction Procedure}

Extraction of the sample spectrum of the buried signal is obtained by decimation. This decimation by the factor $\beta$ is applied in the frequency domain to the Fourier transformation of (3). The signal-to-noise ratio $\hat{\Upsilon}$ of the decimated spectrum written as a function of the signal-to-noise ratio of the original spectrum $\Upsilon$ is given by,

$$
\hat{\Upsilon}=\beta \Upsilon \text {. }
$$

We have shown in [12] that increasing $\beta$ increases the signal-to-noise ratio $\Upsilon$ of the original noisy spectrum $\Phi\left(f, f_{e}, T\right)$ in which the desired signal is buried. Moreover, the variance of sample spectral estimates of noise tends to zero as $\beta$ increases.

\section{Non-Ergodic Processes}

Here, we extend above results obtained for ergodic stationary processes to processes for which ergodic hypothesis cannot be validated or satisfied. We consider therefore that we have a collection of $\beta$ sequences whose probability density functions that describe noise affecting them are different from sequence to an other one. This means that any sample process can be put under the form,

$$
\mu_{T}^{(p)}(t)=s_{T}(t)+b_{T}^{(p)}(t),
$$

where $s_{T}(t)$ is the signal defined above and $b_{T}^{(p)}(t)$ is the additive noise specified for the realization $(p)$. Moreover, probability density functions describing $b_{T}^{(p)}(t)$ for $p=0, \cdots, \beta-1$ are assumed unknown.

Clearly the process from which samples $\mu_{T}^{(p)}(t)$ are taken is stationary and not ergodic. Covariances of the $\beta$ sample processes $\mu_{T}^{(p)}(t)$ are dependent on the sample process $(p)$ (see p. 89 of [19], for the definition of non-ergodic processes). For implementation simplicity, we use hereafter the CFED method, recalled above, where those $\beta$ samples of a non-ergodic process are concatenated, i.e.,

$$
\mu_{\beta T}^{(p)}(t)=\sum_{p=0}^{\beta-1}\left[s_{T}(t-p T)+b_{T}^{(p)}(t-p T)\right] .
$$

\subsection{CFED Sample Spectrum}

The sample spectrum of the process of duration $\beta T$, as depicted by (10), is given by,

$$
\begin{aligned}
\Phi\left(f, f_{e}, \beta T\right) & =\Gamma\left(f, f_{e}, \beta T\right)+\phi\left(f, f_{e}, \beta T\right) \\
& +S^{*}\left(f, f_{e}, \beta T\right) \varphi\left(f, f_{e}, \beta T\right) \\
& +S\left(f, f_{e}, \beta T\right) \varphi^{*}\left(f, f_{e}, \beta T\right),
\end{aligned}
$$

where $S\left(f, f_{e}, \beta T\right)$ and $\varphi\left(f, f_{e}, \beta T\right)$ represent the amplitude spectra of respectively the signal and noise. Here $\varphi^{*}(x)$ is the complex conjugate of $\varphi(x)$ and $\phi\left(f, f_{e}, \beta T\right)$ is the sample spectrum of $\beta$ concatenated realizations of noise.

Now, let us find the optimal form under which expression of the CFED sample spectrum is written only as a function of the sample spectrum of the signal and noise independently of any correlation between the signal and noise and without averaging in the time or frequency domain.

\subsubsection{Sample Spectrum of Noise}

Expression of the sample spectrum of noise of a non-ergodic process is obtained by using the above expression (4) derived under the ergodic assumption. In (4), one finds translated copies of the original sample spectrum of noise, denoted in the ergodic case by $\phi\left(f-p /(\beta T), f_{e}, T\right)$, of each realization or sample process for $p=0, \cdots, \beta-1$.

Now, in the non-ergodic framework, as depicted by (10), it is crucial to notice that we have $\beta$ realizations defined for different and unknown noise distributions. As in (10) the index $(p)$ identifies each noise distribution, here, we introduce the index $(p)$ in order to identify their corresponding $\beta$ original and different sample spectra. We can therefore rewrite (4) by identifying translated copies of original sample spectra of noise by their corresponding upper script $(p)$ (for $p=0, \cdots, \beta-1$ ) as follows,

$$
\phi\left(f, f_{e}, \beta T\right)=\sum_{p=0}^{\beta-1} \alpha_{p}(\beta) \phi_{p}^{(p)}\left(f, f_{e}, T\right),
$$

where,

$$
\phi_{p}^{(p)}\left(f, f_{e}, T\right)=\phi^{(p)}\left(f-p /(\beta T), f_{e}, T\right) .
$$

\subsubsection{Decimated CFED Sample Spectrum}

The decimated $N$-point sample spectrum applied to $\Phi\left(f, f_{e}, \beta T\right)$, as depicted by (11), yields (14). where $\mathcal{D}_{\beta}[\Phi]$ is the $\beta$-decimation applied to $\Phi$.

Notice that since the power spectral density of the signal is assumed constant in collected $\beta$ sequences then decimated sample spectrum of the signal yields the same

$$
\begin{aligned}
\mathcal{D}_{\beta}\left[\Phi\left(f, f_{e}, \beta T\right)\right] & =\mathcal{D}_{\beta}\left[\Gamma\left(f, f_{e}, \beta T\right)\right]+\mathcal{D}_{\beta}\left[\phi\left(f, f_{e}, \beta T\right)\right] \\
& +\mathcal{D}_{\beta}\left[S^{*}\left(f, f_{e}, \beta T\right) \varphi\left(f, f_{e}, \beta T\right)+S\left(f, f_{e}, \beta T\right) \varphi^{*}\left(f, f_{e}, \beta T\right)\right],
\end{aligned}
$$


result as (7).

On the other hand, since we have $\beta$ translated and different copies of original sample spectra of noise then decimation in the frequency domain yields a spectrum in which contribute coefficients of those $\beta$ copies of original spectra of noise. This means that the $N$ coeffi- cients of the decimated spectrum described by (12) are those of the translated copies $\phi^{(p)}\left(f-p /(\beta T), f_{e}, T\right)$ for $p=0, \cdots, \beta-1$. Notice that this applies also to the amplitude spectra of noise $\varphi\left(f, f_{e}, \beta T\right)$. This means that by setting $\alpha_{p}(\beta)=1 / \beta$, the decimated sample spectrum of noise in (14) becomes,

$$
\begin{aligned}
\mathcal{D}_{\beta}\left[\phi\left(f, f_{e}, \beta T\right)\right] & =\mathcal{D}_{\beta}\left[\sum_{p=0}^{\beta-1} \alpha_{p}(\beta) \phi^{(p)}\left(f-p /(\beta T), f_{e}, T\right)\right] \\
& =(1 / \beta) \phi\left(f, f_{e}, T ; \phi^{(0)}, \cdots, \phi^{(\beta-1)}\right),
\end{aligned}
$$

where the set of the copies $\left\{\phi^{(0)}, \cdots, \phi^{(\beta-1)}\right\}$ is given by $\phi^{(p)}\left(f-p /(\beta T), f_{e}, T\right)$ for $p=0, \cdots, \beta-1$.

Since Fourier coefficients of $\phi\left(f, f_{e}, T ; \phi^{(0)}, \cdots, \phi^{(\beta-1)}\right)$ are those of the translated copies $\phi^{(0)}, \cdots, \phi^{(\beta-1)}$ then the noise variance $\sigma_{\phi}^{2}$ of $\phi\left(f, f_{e}, T ; \phi^{(0)}, \cdots, \phi^{(\beta-1)}\right)$ is given by,

$$
\sigma_{\min }^{2}<\sigma_{\varphi}^{2}<\sigma_{\max }^{2},
$$

where $\sigma_{\min }^{2}$ and $\sigma_{\max }^{2}$ are respectively the smallest and the greatest noise variances contained in the collection of $\beta$ sequences.

It is crucial to notice that (16) is at the heart of this work. According to (16), we can assume, for the sake of simplicity and without loss of generality, that the decimated sample spectrum of noise $\phi\left(f, f_{e}, T ; \phi^{(0)}, \cdots, \phi^{(\beta-1)}\right)$ approaches the average sample spectra of the translated copies $\phi^{(0)}, \cdots, \phi^{(\beta-1)}$ of noise. Under this assumption, justified by (16), we can write the variance of the decimated sample spectrum of noise $\phi\left(f, f_{e}, T ; \phi^{(0)}, \cdots, \phi^{(\beta-1)}\right)$ as follows,

$$
\sigma_{\varphi}^{2}=\bar{\sigma}^{2}=(1 / \beta) \sum_{k=0}^{N-1 \beta-1} \sum_{p=0}^{(p)} c_{k},
$$

where $c_{k}^{(p)}$ for $k=0 \cdots N-1$ and $p=0 \cdots \beta-1$ are Fourier coefficients of the translated copies $\phi^{(0)}, \cdots, \phi^{(\beta-1)}$.

Now, according to (17), the sample spectrum $\phi\left(f, f_{e}, T ; \phi^{(0)}, \cdots, \phi^{(\beta-1)}\right)$ written as a function of its Fourier coefficients, yields,

$$
\phi\left(f, f_{e}, T ; \phi^{(0)}, \cdots, \phi^{(\beta-1)}\right)=\sum_{k=0}^{N-1} \bar{c}_{k} \delta(f-k / T),
$$

where $\bar{c}_{k}=\left(c_{k}^{(0)}+\cdots+c_{k}^{(\beta-1)}\right) / \beta$ represents the average of coefficients of translated copies $\phi^{(0)}, \cdots, \phi^{(\beta-1)}$. Notice that the ergodic case $[12,13]$ can be obtained from (18) by setting $c_{k}^{(0)}=\cdots=c_{k}^{(\beta-1)}$.

By using (7) and (15), the decimated sample spectrum of the process, as given by (14), is therefore given by,

$$
\begin{aligned}
& D_{\beta}\left[\Phi\left(f, f_{e}, \beta T\right)\right]=\Gamma\left(f, f_{e}, T\right)+(1 / \beta) \phi\left(f, f_{e}, T ; \phi^{(0)}, \cdots, \phi^{(\beta-1)}\right) \\
& \quad+S^{*}\left(f, f_{e}, T\right) \varphi\left(f, f_{e}, T ; \phi^{(0)}, \cdots, \phi^{(\beta-1)}\right)+S\left(f, f_{e}, T\right) \varphi^{*}\left(f, f_{e}, T ; \phi^{(0)}, \cdots, \phi^{(\beta-1)}\right),
\end{aligned}
$$

where decimated power spectrum of noise written as a function of its amplitude spectra yields,

$$
\phi\left(f, f_{e}, T ; \phi^{(0)}, \cdots, \phi^{\beta-1}\right) / \beta=\varphi\left(f, f_{e}, T ; \phi^{(0)}, \cdots, \phi^{(\beta-1)}\right) \times \varphi^{*}\left(f, f_{e}, T ; \phi^{(0)}, \cdots, \phi^{(\beta-1)}\right) .
$$

\subsection{Optimal CFED Sample Spectrum}

Let us in the following find a condition under which the decimated sample spectrum as depicted by (19) can be written without rectangular terms (cross-products). The optimal form is that for which the sample spectrum is described only as function of the spectra of the signal and noise.

Let $\lambda_{k}$ be Fourier coefficients of the amplitude spectrum $S\left(f, f_{e}, T\right)$ of the signal and let $\bar{\xi}_{k}$ be Fourier coefficients of the amplitude noise spectrum $\varphi\left(f, f_{e}, T ; \phi^{(0)}, \cdots, \phi^{(\beta-1)}\right)$. Since,

$$
\Gamma\left(f, f_{e}, T\right)=S\left(f, f_{e}, T\right) S^{*}\left(f, f_{e}, T\right),
$$

then according to (20) and (21), we have,

$$
\begin{aligned}
& \gamma_{k}=\lambda_{k} \lambda_{k}^{*} \\
& \bar{c}_{k} / \beta=\bar{\xi}_{k}\left(\bar{\xi}_{k}\right)^{*},
\end{aligned}
$$

where $\bar{c}_{k}$ is defined in (18).

By using (22), the sample spectrum, as given by (19), yields therefore explicitly,

$$
\begin{gathered}
\mathcal{D}_{\beta}\left[\Phi\left(f, f_{e}, \beta T\right)\right]=(1 / \beta) \phi\left(f, f_{e}, T ; \phi^{(0)}, \cdots, \phi^{\beta-1}\right) \\
+\sum_{k=0}^{N-1}\left[\gamma_{k}+\lambda_{k}\left(\bar{\xi}_{k}\right)^{*}+\lambda_{k}^{*} \bar{\xi}_{k}\right] \delta(f-k / T) .
\end{gathered}
$$

\subsubsection{The Optimal Reduction Factor}

In the following, we derive the optimal reduction factor or the optimal number of concatenated sample processes 
$\beta$ under which contribution of the cross-products in (19) is made negligible. Coefficients of the last right-hand side of (23) can be put under the form,

$$
\gamma_{k}+\lambda_{k}\left(\bar{\xi}_{k}\right)^{*}+\lambda_{k}^{*} \bar{\xi}_{k}=\gamma_{k} \times\left[1+\left(\frac{\bar{\xi}_{k}}{\lambda_{k}}\right)^{*}+\frac{\bar{\xi}_{k}}{\lambda_{k}}\right]
$$

Let $\psi_{k}=\bar{\xi}_{k} / \lambda_{k}+\left(\bar{\xi}_{k} / \lambda_{k}\right)^{*}$ and note that,

$$
\left|1+\psi_{k}\right| \leq 1+\left|\psi_{k}\right|
$$

where $\psi_{k}$ rewritten as a function of $\bar{\xi}_{k}$ and $\lambda_{k}$ yields,

$$
\left|\frac{\bar{\xi}_{k}}{\lambda_{k}}+\left(\frac{\bar{\xi}_{k}}{\lambda_{k}}\right)^{*}\right| \leq 2 \frac{\left|\bar{\xi}_{k}\right|}{\left|\lambda_{k}\right|}
$$

By setting $\bar{c}_{k} / \beta=\bar{\xi}_{k}\left(\bar{\xi}_{k}\right)^{*}$, (26) becomes,

$$
\left|\frac{\bar{\xi}_{k}}{\lambda_{k}}+\left(\frac{\bar{\xi}_{k}}{\lambda_{k}}\right)^{*}\right| \leq 2 \sqrt{\frac{\bar{c}_{k}}{\beta \gamma_{k}}}
$$

Now, let us find the condition that defines the minimum value of $\beta$ under which (27) is smaller than unity, i.e.,

$$
2 \sqrt{\frac{\bar{c}_{k}}{\beta \gamma_{k}}} \ll 1
$$

We propose to find $\beta$ as a function of the mean signal-to-noise ratio of the $\beta$ collection of different processes. Let us define the mean signal-to-noise ratio of the collected $\beta$ sample processes by,

$$
\bar{\Upsilon}=p_{s} / \bar{\sigma}^{2},
$$

where $p_{s}$ represents the mean power of the signal and $\bar{\sigma}^{2}=\left(\sigma_{(0)}^{2}+\cdots+\sigma_{(\beta-1)}^{2}\right) / \beta$ is the mean variance of noise of the collected $\beta$ sample processes. Here $\sigma_{(p)}^{2}$ is noise variance of the sample process $(p)$ (for $p=0, \cdots, \beta-1)$.

The mean signal-to-noise ratio can be written under the form,

$$
\begin{aligned}
\bar{\Upsilon} & =p_{s} / \bar{\sigma}^{2} \\
& =\frac{\gamma_{k}}{\bar{c}_{k}} \times \frac{I_{\gamma}}{I_{c}},
\end{aligned}
$$

where $\gamma_{k}$ and $\bar{c}_{k}$ are arbitrary chosen coefficients and,

$$
\begin{aligned}
& I_{\gamma}=1+\sum_{s=0, s \neq k}^{\mathcal{S}-1} \gamma_{s} / \gamma_{k} \\
& I_{c}=1+\sum_{q=0, q \neq k}^{\mathcal{N}-1} \bar{c}_{q} / \bar{C}_{k},
\end{aligned}
$$

where $\mathcal{S}$ and $\mathcal{N}$ represent respectively the number of components of the signal and noise.

It is easy to see that $I_{\gamma}$ is bounded by,

$$
\forall k, \mathcal{S} \min \left\{\gamma_{s} / \gamma_{k}\right\} \leq I_{\gamma} \leq \mathcal{S} \max \left\{\gamma_{s} / \gamma_{k}\right\},
$$

where $\min \left\{\gamma_{s} / \gamma_{k}\right\}$ and $\max \left\{\gamma_{s} / \gamma_{k}\right\}$ denote respectively the minimum and the maximum values of the set formed by $\gamma_{s} / \gamma_{k}$, for $s=0,1, \cdots, \mathcal{S}-1$ and $\forall k$.

Since $\gamma_{k}$ is an arbitrary chosen coefficient and according to (31), we can consider that,

$$
\forall k, I_{\gamma}=\mathcal{S} \text {. }
$$

Similarly, $I_{c}=\mathcal{N}$. The signal-to-noise ratio, as depicted by (29), becomes,

$$
\bar{\Upsilon}=\frac{\gamma_{k}}{\bar{c}_{k}} \frac{\mathcal{S}}{\mathcal{N}} .
$$

Now, the expression (28) is satisfied if,

$$
\beta \gg \frac{4 \mathcal{S}}{\mathcal{N} \bar{\Upsilon}} \text {. }
$$

For a useful interpretation of (34), we express the optimal reduction factor $\beta$ only as a function of the mean signal-to-noise ratio $\bar{\Upsilon}$. By setting $\beta_{\text {min }}=4 \mathcal{S} /(\mathcal{N} \bar{\Upsilon})$, one finds that since $\mathcal{S} / \mathcal{N}<1$ two conditions have to be considered : $4 \mathcal{S} / \mathcal{N} \leq 1$ and $4 \mathcal{S} / \mathcal{N} \geq 1$. This gives,

$$
\begin{aligned}
& 4 \mathcal{S} / \mathcal{N} \leq 1, \quad 1<\beta_{\min } \leq \frac{1}{\bar{\Upsilon}} \\
& 4 \mathcal{S} / \mathcal{N} \geq 1, \quad \beta_{\min } \geq \frac{1}{\bar{\Upsilon}} .
\end{aligned}
$$

As $\beta \gg \beta_{\text {min }}$ in accordance with (34), then by choosing $\beta$ exceeding the upper bound of the variation interval of $\beta_{\text {min }}$, or,

$$
\beta>\frac{1}{\bar{\Upsilon}}
$$

the condition (28) is fulfilled.

Here (36) depicts the optimal reduction factor $\beta$ as a function of the mean signal-to-noise ratio $\bar{\Upsilon}$ of the collection of $\beta$ non-ergodic sample processes. This optimal reduction factor represents therefore the number of concatenated sample processes.

\subsubsection{Optimal CFED Spectrum}

According to (36), (24) yields,

$$
\forall \beta>1 / \bar{\Upsilon},\left|\gamma_{k}+\lambda_{k} \bar{\zeta}_{k}^{*}+\lambda_{k}^{*} \bar{\zeta}_{k}\right| \leq \gamma_{k}
$$

By using (37), (23) becomes,

$$
\begin{gathered}
\forall \beta>1 / \bar{\Upsilon}, \mathcal{D}_{\beta}\left[\Phi\left(f, f_{e}, \beta T\right)\right] \leq \Gamma\left(f, f_{e}, T\right) \\
+\frac{1}{\beta} \phi\left(f, f_{e}, T ; \phi^{(0)}, \cdots, \phi^{(\beta-1)}\right) .
\end{gathered}
$$

This yields, 


$$
\begin{aligned}
& \forall \beta>1 / \bar{\Upsilon}, \mathcal{D}_{\beta}\left[\Phi\left(f, f_{e}, \beta T\right)\right]=\Phi_{\max }\left(f, f_{e}, T\right) \\
& =\Gamma\left(f, f_{e}, T\right)+\frac{1}{\beta} \phi\left(f, f_{e}, T ; \phi^{(0)}, \cdots, \phi^{(\beta-1)}\right) .
\end{aligned}
$$

Here $\beta>1 / \bar{\Upsilon}$ represents the number of concatenated sample processes under which the decimated sample spectrum of the process becomes optimal or be written under the form (39).

It can be seen that under the condition (36) contribution of the cross-products is made negligible. Expression of the extracted spectrum of the buried signal from non-ergodic processes, as depicted by (39), can be obtained without the requirement based on ensemble averaging and without assuming the signal uncorrelated with noise. Moreover, at the limit of large values of $\beta$, (39) becomes,

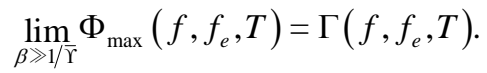

The sample spectrum of noise, independently of its nature, vanishes. The decimated spectrum is identical to the original deterministic spectrum of the signal. Results (40) and (39) achieve extraction of buried spectra of signals from non-ergodic processes.

\section{Method and Results}

\subsection{Experimental Signals: Doppler Velocimetry}

It is well known that Ultrasonic Doppler velocimetry provides a non-invasive method for measuring direction and speed of fluids. Information of interest on Doppler signals may be found, for example, in [6-11]. Signals used here are issued from a flow measurement apparatus that uses pulsated emitting source with a constant Pulse Repetition Frequency (sampling frequency) PRF $=83.3$ $\mathrm{kHz}$. A fluid runs at constant or quasi-constant speed and the concern here is to measure its mean speed [6,9]. The Doppler mean frequency $\Delta f$ is related to the mean velocity $v$ of the flow by (see [10] among above references),

$$
\Delta f=2 v \cos (\theta) v / c,
$$

where $v$ is the frequency of the pulsated emitting source, $c$ is the velocity of the ultrasound wave and $\theta$ defines the receiver position with respect to the direction of the flow. Here $v=2 \times 10^{6} \mathrm{~Hz}, c=1500 \mathrm{~m} / \mathrm{s}$ and $\theta=\pi / 4$. In this experiment, the mean velocity of the flow is $v=4 \mathrm{~m} / \mathrm{s}$. The expected Doppler mean frequency is therefore $\Delta f=7.54 \mathrm{kHz}$.

\subsection{Non-Ergodicity of Doppler Sequences}

In Figure 1, zero-mean four realizations (a1)-(a4) of
Doppler velocimetry signals carrying information on the speed of the fluid are depicted. Here we assume that the nature of noise (white or colored, correlated or not) affecting the Doppler mean frequency is unknown.

Moreover, to accentuate non-ergodicity effect, let us add to the first Doppler sequence, (a1), the third one, (a3), and the last one (a4), three different colored noise sequences $y_{a 1}(n), y_{a 3}(n)$ and $y_{a 4}(n)$ specified by,

$$
\begin{aligned}
y_{a 1}(n)= & -(1.4+0.5 i) y_{a 1}(n-1) \\
& -(0.45+0.45 i) y_{a 1}(n-2)+e(n) \\
y_{a 3}(n)= & 0.89 y_{a 3}(n-1)-0.65 y_{a 3}(n-2) \\
& +0.38 u(n-1)+0.38 u(n)+e(n) \\
y_{a 4}(n)= & -0.9 y_{a 4}(n-2)+0.89 y_{a 4}(n-1) \\
& +0.19 e(n-2)+0.38 e(n-1)+0.2 e(n),
\end{aligned}
$$

where $u(n)$ is a random signum function (logical function which extracts the sign of a uniformly distributed random number) and $e(n)$ is white Gaussian noise sequence. The length of these sequences is $N$.

We assume that signal-to-noise ratios of these Doppler sequences are unknown. However, mean powers of collected Doppler sequences can be computed. These are variances of noise since the Doppler signal carrying the Doppler mean frequency is very weak. We have $\sigma_{a 1}^{2}=1.3(1.14 \mathrm{~dB}), \quad \sigma_{a 2}^{2}=0.6(-2.2 \mathrm{~dB}), \quad \sigma_{a 3}^{2}=0.7$ $(-1.55 \mathrm{~dB})$ and $\sigma_{a 4}^{2}=1.46(1.5 \mathrm{~dB})$. This creates variations of the signal-to-noise ratio. According to (39), we have $\beta \bar{\Upsilon}>1$. Since $\beta=4$, the mean signal-to-noise ratio for which an extraction from noise is possible is $\bar{\Upsilon}>0.25(-6 \mathrm{~dB})$. If no extraction is obtained, this means that the mean signal-to-noise ratio of Doppler sequences is lower than $-6 \mathrm{~dB}$.

In Figure 1, histograms (b1), (b2), (b3) and (b4) of the four Doppler sequences are shown. It can be seen that we have four different and deformed noise distributions with varying amplitudes. In (c1), (c2), (c3) and (c4), covariance functions of the Doppler sequences are depicted in logarithmic scales for better visibility (low correlation lags) and for comparison purposes. These covariance functions are clearly dependent on the sample Doppler process. We have therefore non-ergodic Doppler velocimetry measurements (see p. 89 of [19]).

\subsection{Extraction Results}

In Figure 2, we propose extraction of the Doppler mean frequency by using CFED method, Welch PSD estimation [15] and the Thomson's multi-window method (MTM) [17]. We recall that MTM uses a bank of windows that compute several periodograms of the entire signal and then averaging the resulting periodograms to 


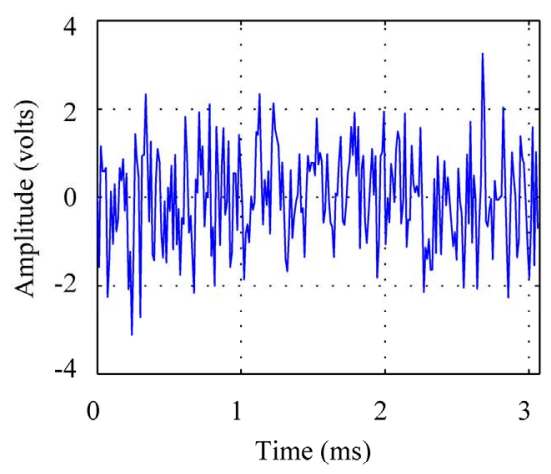

(a1)

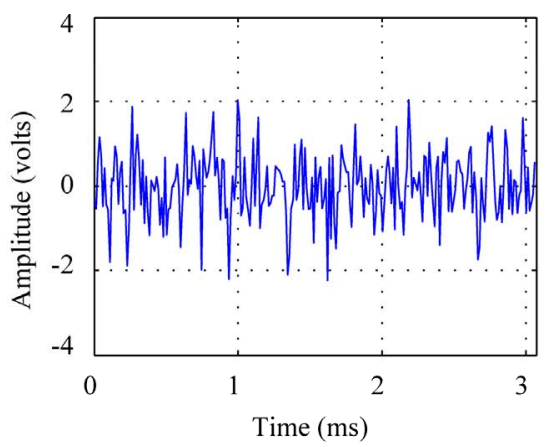

(a2)

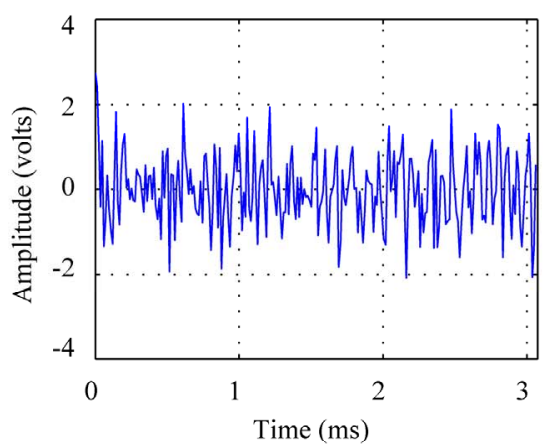

(a3)

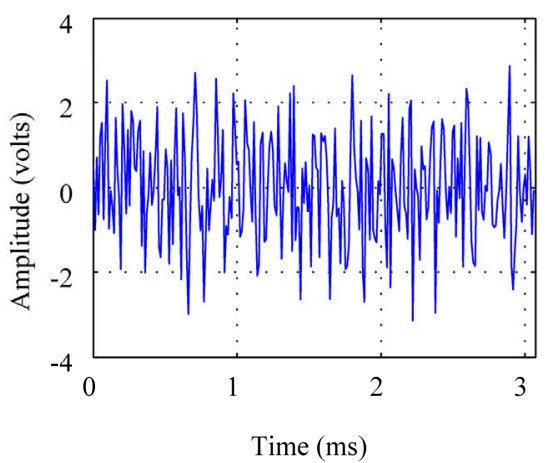

(a4)

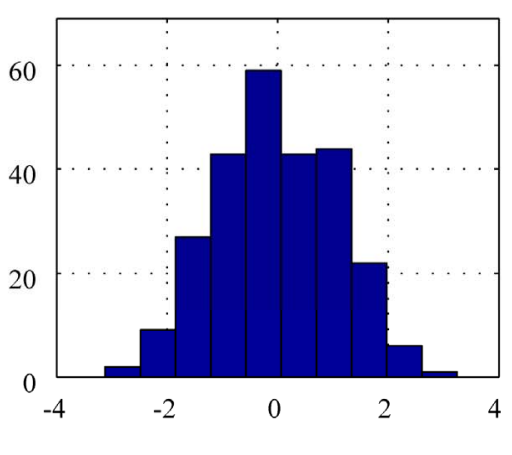

(b1)

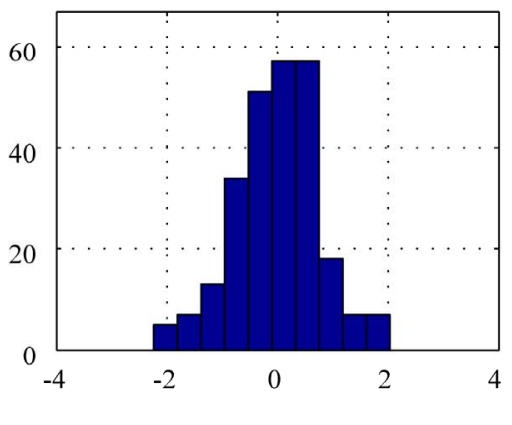

(b2)

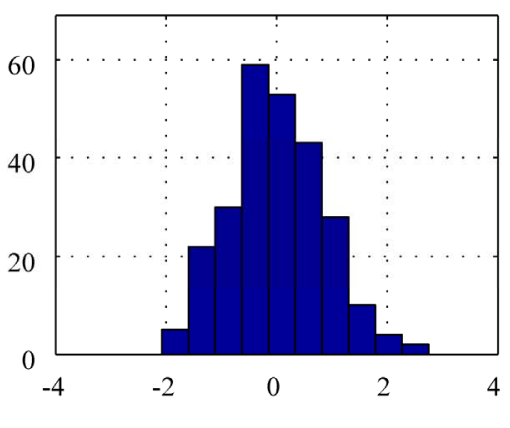

(b3)

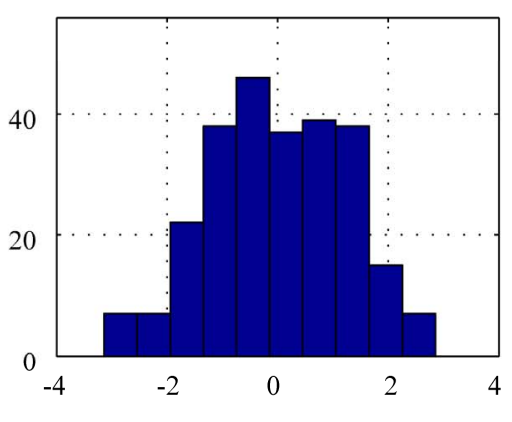

(b4)

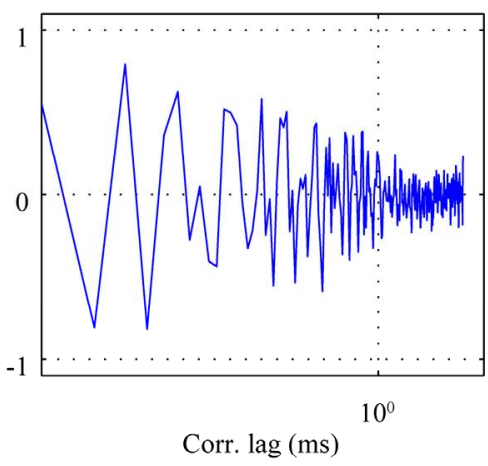

(c1)

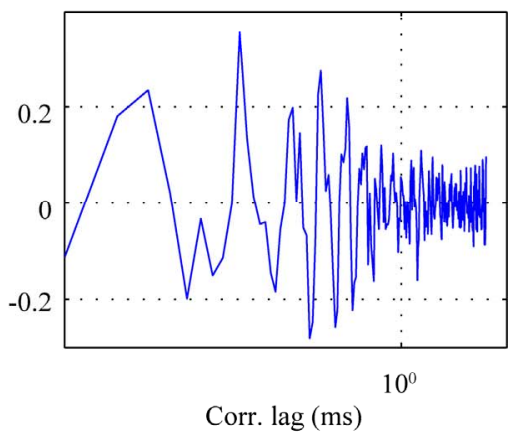

(c2)

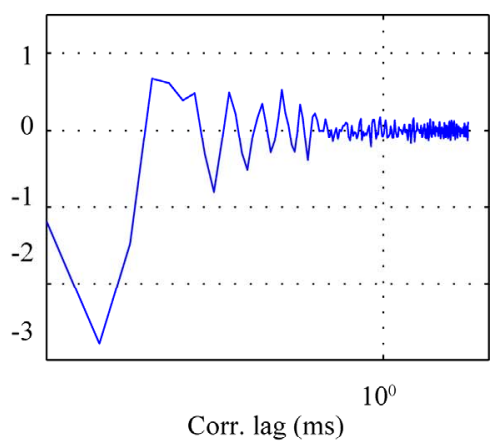

(c3)

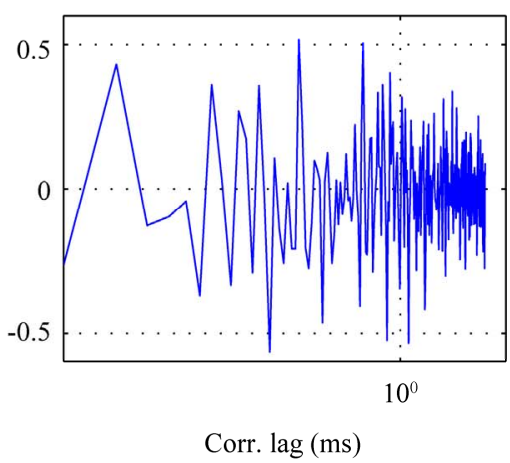

(c4)

Figure 1. Four Doppler velocimetry measurements (a1), (a2), (a3) and (a4) in which the Doppler mean frequency is buried in white and colored noise. Non-ergodic character is shown by different histograms and corresponding different covariance functions (in logarithmic scale). 


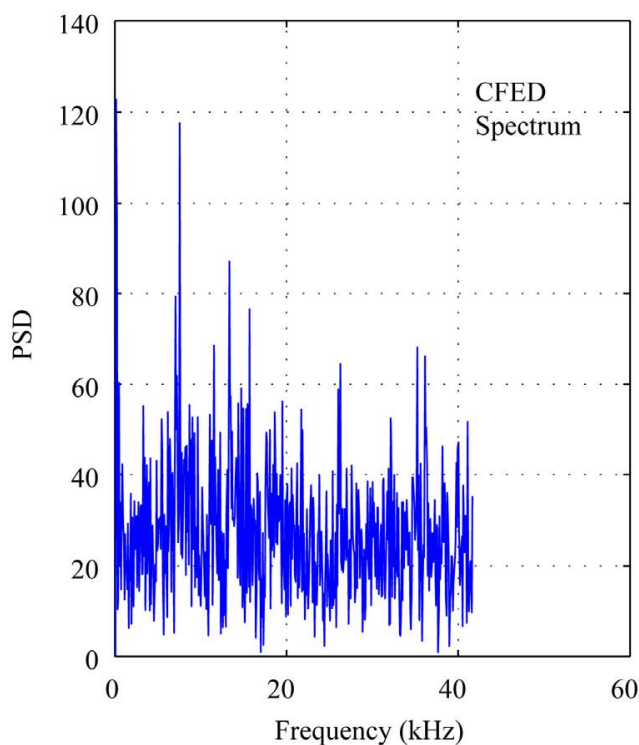

(a)

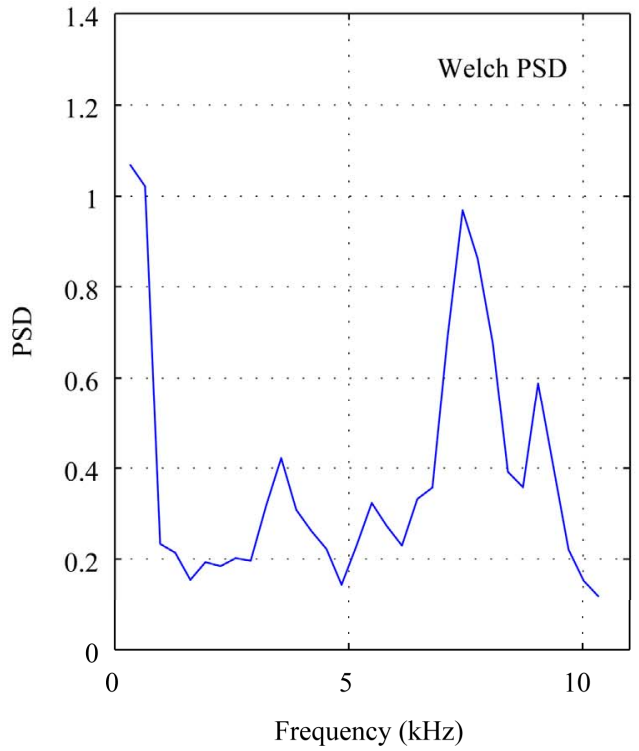

(c)

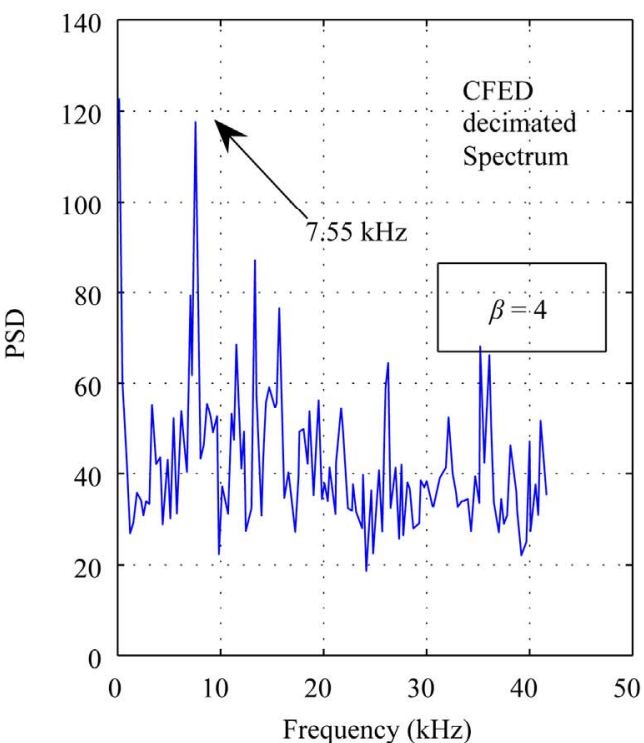

(b)

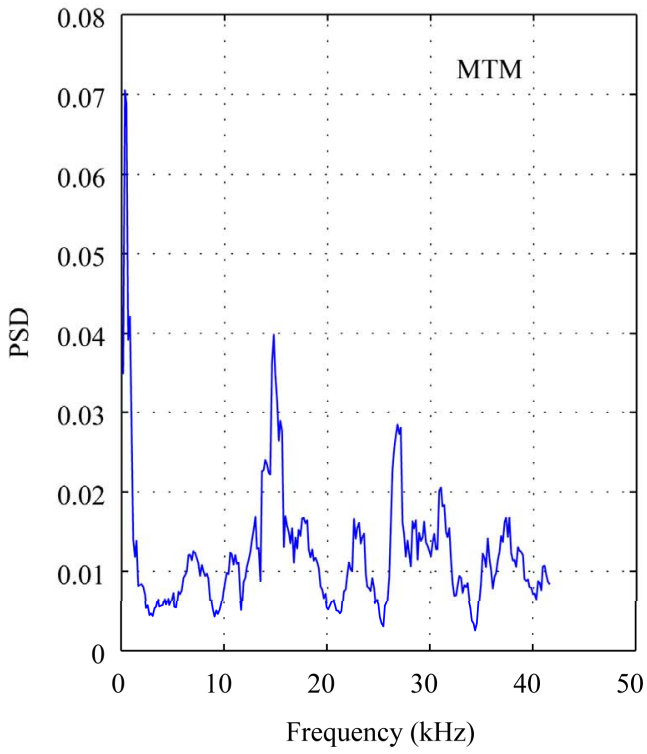

(d)

Figure 2. CFED spectrum and its decimated version are shown in (a) and (b) where the Doppler frequency is extracted (7.55 kHz). Comparison with Welch PSD and the Thomson's multitaper method is provided in (c) and (d).

construct a spectral estimate. In order to minimize the bias and variance in each window, theses windows are chosen orthogonal. Optimal windows that satisfy these requirements are Slepian sequences or discrete prolate spheroidal sequences [18].

Now, these four realizations $(\beta=4)$ are concatenated and the obtained process, as given by (10), is analyzed by the above methods. The spectrum of CFED concatenated realizations and its decimated version $(\beta=4)$ are respectively shown in Figures 2(a) and (b). The frequency of the depicted spectral line (Doppler mean frequency) is $\hat{\Delta} f=7.55 \mathrm{kHz}$. The obtained varia- tion with respect to the above expected Doppler frequency is $0.13 \%$. The Welch periodogram estimation shows however a widened peak located at $7.4 \mathrm{kHz}$ with a weak signal-to-noise ratio whereas the MTM method depicts with a much weaker signal-to-noise ratio a spectral line far from the expected Doppler mean frequency.

It can be seen that independently of the nature of noise (white or colored, correlated or not) affecting experimental signals and variation of the signal-to-noise ratio, the Doppler mean frequency is clearly extracted by CFED from non-ergodic process with an excellent signal-to-noise ratio without any averaging in the time or 
frequency domain and without using any a-priori information on the signal (Doppler mean frequency) and the nature of noise in which the signal is buried.

\section{Conclusions}

In this work, extraction theory of signals buried in non-ergodic processes is proposed. We have shown that no a-priori information on the signal to be extracted is used and no averaging in the direct time or frequency domain is performed. Observed results on experimental Doppler velocimetry measurements buried in non-ergodic processes show that extraction of the Doppler mean frequency is achieved independently of the nature of noise, correlated or not with the signal, colored or white, Gaussian or not, and locations of its spectral extent. Observed results are in accordance with theoretical predictions.

\section{Acknowledgements}

The author wishes to express his thanks to anonymous reviewers for their constructive suggestions.

\section{References}

[1] J. L. Starck and F. Murtagh, "Astronomical Image and Data Analysis,” Springer Verlag Inc., New York, 2006.

[2] P. N. Pusey and W. van Megen, "Dynamic Light Scattering by Non-Ergodic Media,” Royal Signals and Radar Establishment, Physica A, Vol. 157, No. 2, 1989, pp. 705-741.

[3] A. Budhiraja and D. Ocone, "Exponential Stability in Discrete Time Filtering for Non-Ergodic Signals," Stochastic Processes and Their Applications, Vol. 82, No. 2, 1999, pp. 245-257.

[4] N. Oudjane and S. Rubenthaler, "Stability and Uniform Particle Approximation of Nonlinear Filters in Case of Non Ergodic Signals," Stochastic Analysis and Applications, Vol. 23, No. 3, 2005, pp. 421-448.

[5] G. Storvik, "Particle Filters for State Space Models with the Presence of Unknown Static Parameters," IEEE Transactions on Signal Processing, Vol. 50, No. 2, 2002, pp. 281-289.

[6] W. D. Barber, J. W. Eberhard and S. G. Kaar, “A New Time-Domain Technique for Velocity Measurements Using Doppler Ultrasound," IEEE Transactions on Biomedical Engineering, Vol. BME-32, No. 3, 1985, pp. 213229.
[7] P. J. Vaitkus and R. S. C. Cobbold, “A Comparative Study and Assessment of Doppler Ultrasound Spectral Estimation Techniques Part I: Estimation Methods,” Ultrasound in Medicine and Biology, Vol. 14, No. 8, 1988 , pp. 661-672.

[8] P. J. Vaitkus, R. S. C. Cobbold and K. W. Johnston, “A Comparative Study and Assessment of Doppler Ultrasound Spectral Estimation Techniques Part II: Method and Results," Ultrasound in Medicine and Biology, Vol. 14, No. 8, 1988, pp. 673-688.

[9] D. H. Evans, W. N. MacDicken, R. Skidmore and J. P. Woodcock, "Summary of the Basic Physics of Doppler Ultrasound," Doppler Ultrasound, Physics Instrumentation and Clinical Applications, Wiley, Chichester, 1989.

[10] J. Arendt, "Estimation of Blood Velocities Using Ultrasound,” Cambridge University Press, Cambridge, 1996.

[11] A. Herment and J. F. Giovanelli, “An Adaptive Approach to Computing the Spectrum and Mean Frequency OD Doppler Signal,” Ultrasonic Imaging, Vol. 17, No. 1, 1995, pp. 1-26.

[12] N. Y. Bey, "Extraction of Signals Buried in Noise, Part I: Fundamentals,” Signal Processing, Vol. 86, No. 9, 2006, pp. 2464-2478.

[13] N. Y. Bey, "Extraction of Signals Buried in Noise, Part II: Experimental Results,” Signal Processing, Vol. 86, No. 10, 2006, pp. 2994-3011.

[14] N. Y. Bey, "Extraction of Buried Signals in Noise: Correlated Processes," International Journal of Communications, Network and System Sciences, Vol. 3, No. 11, 2010, pp. 855-862.

[15] P. D. Welch, "The Use of Fast Fourier Transform for the Estimation of Power Spectra: A Method Based on Time Averaging over Short, Modified Periodograms," IEEE Transactions on Audio and Electroacoustics, Vol. AU-15, 1967, pp. 70-73.

[16] S. M. Kay and S. L. Marple, "Spectrum Analysis-A Modern Perspective," Proceedings of IEEE, Vol. 69, No. 11, 1981, pp. 1380-1419.

[17] D. J. Thomson, "Spectrum Estimation and Harmonic Analysis,” Proceedings of the IEEE, Vol. 70, No. 9, 1982, pp. 1055-1096.

[18] Y. Xu, S. Haykin and R. J. Racine, "Multiple Window Time-Frequency Distribution and Coherence of EEG Using Slepian Sequences and Hermite Functions," IEEE Transactions on Biomedical Engineering, Vol. 46, No. 7, 1999, pp. 861-866.

[19] J. S. Bendat, "Measurement and Analysis of Random Data,” John Wiley and Sons Inc., Hoboken, 1966. 